\title{
Non-destructive characterization of CoTSPc electrochemically deposited on gold electrodes by means of synchrotron X-ray microfluorescence
}

\author{
Karl Peeters ${ }^{\mathrm{a}}$, Karolien De Wael ${ }^{\mathrm{a}, *}$, Annemie Adriaens ${ }^{\mathrm{a}}$, \\ Gerald Falkenberg ${ }^{\mathrm{b}}$, Laszlo Vincze ${ }^{\mathrm{a}}$ \\ a Ghent University, Department of Analytical Chemistry, Krijgslaan 281 S12, B-9000 Ghent, Belgium \\ ${ }^{\mathrm{b}}$ Hamburger Synchrotronstrahlungslabor at Deutsches Elektronen-Synchrotron, DESY, D-22607 Hamburg, Germany
}

Received 5 July 2005; received in revised form 10 August 2005; accepted 16 August 2005

Available online 21 September 2005

\begin{abstract}
This paper gives a detailed study of the morphology and heterogeneity of an electrodeposited cobalt tetrasulphophthalocyanine tetrasodium salt (Co(II)TSPc) layer. A continuous potential cycling of the electrode between two potentials can lead to a modification of a bare electrode. During the modification, a non-destructive characterization by means of scanning synchrotron X-ray fluorescence radiation has been made as a function of scan number. Moreover, electrochemical and SR-XRF measurements were performed in order to calculate the adsorbed Co surface concentration and to distinguish between monomer and dimer adsorption.
\end{abstract}

(C) 2005 Elsevier B.V. All rights reserved.

Keywords: Gold; Electrochemical modification; Phthalocyanines; Morphology; Synchrotron X-ray fluorescence

\section{Introduction}

Transition metallo phthalocyanines have lately attracted a wide research interest, including that of electrochemists. They are commonly used in high-tech applications, such as photosensitizers, in optical data storage and chemical gas sensors [1-5]. The use of phthalocyanines has been expanding into other applied fields, e.g., they are frequently used molecules in electrocatalysis [6-8]. Their major characteristics are their high thermal and chemical stability, coupled with their extensive redox chemistry.

Soluble sulphonated phthalocyanines have been shown to be able to form layers at different types of supporting material, including electrodes. The immobilization of these compounds on an electrode can lead to modified electrodes

\footnotetext{
* Corresponding author. Postdoctoral Fellow of the Research Foundation - Flanders (FWO - Vlaanderen). Tel.: +329 26448 20; fax: +329264 4960 .

E-mail address: Karolien.DeWael@UGent.be (K. De Wael).
}

with electrocatalytic properties. More than 70 different metal ions can form a complex by coordinating with the phthalocyanine ring [1]. This aromatic ring structure is completely flat which enables the phthalocyanines in the deposited layer to form columnar aggregates, in which $\pi$ stacking interactions between the molecules dominate. It is desirable to have ordered, well-packed and well-oriented molecular layers of phthalocyanines on the electrodes. The aromatic ring structure can be oxidised and/or reduced. If the central metal ion is a transition element, it can undergo oxidation or reduction at potentials comparable to those of the ring processes [1].

Various techniques have been used to create modified electrodes, including drop drying, spin coating and spontaneous adsorption (self-assembling) [9-11]. In comparison with these techniques, electrodeposition is a technique that enables one to control and to characterize the formed layer by following the potential and current during the process. In this study, this technique has been used to deposit a film of phthalocyanines onto a gold 
electrode. A continuous cycling of the electrode between two potentials in a phthalocyanine solution can lead to a modification of the original electrode surface. By measuring the current as a function of potential and applied number of cycles, it is possible to follow the adsorption process in situ. In previous articles of the authors, the deposition of cobalt(II) tetrasulphonated phthalocyanine (CoTSPc) on gold surfaces in alkaline solution is well described [12,13]. According to the authors, a detailed study of the morphology and heterogeneity of the deposited phthalocyanine layer as a function of scan number has not yet been described.

This paper describes the non-destructive characterization of CoTSPc thin films electrochemically deposited on gold electrodes as a function of scan number by means of synchrotron X-ray micro fluorescence (SR-XRF).

\section{Experimental}

The CoTSPc deposition on gold electrodes was performed using an electrochemical cell equipped with a saturated calomel reference electrode (SCE) with two compartments from Radiometer (Analis, Belgium) and a carbon counter electrode. The working electrodes were gold electrodes from BAS (Bioanalytical Systems Inc., USA), having a diameter of $1.6 \mathrm{~mm}$, which were pretreated by mechanical and electrochemical polishing. Before its first use the electrode surface was scoured briefly on SiC-emery paper 1200 grit to obtain a fresh surface. In order to reduce the relatively high surface roughness the electrode was further subjected to sequential polishing using a polishing cloth covered with alumina powder (Buehler) of 1, 0.3 and $0.05 \mu \mathrm{m}$ particle size for, respectively 5, 10 and 20 min. To remove any adherent $\mathrm{Al}_{2} \mathrm{O}_{3}$ particles the electrode surface was rinsed thoroughly with doubly deionised water and cleaned in an ultrasonic bath (Branson 3210) for $2 \mathrm{~min}$. Finally, the electrode was pretreated electrochemically by scanning it in a $\mathrm{Na}_{2} \mathrm{HPO}_{4} / \mathrm{NaOH}$ buffer solution ( $\mathrm{pH}=12$, purchased from Riedel-de Haën, Belgium) between -1.2 and $0.6 \mathrm{~V}$ vs. SCE until five subsequent scans were identical. This pretreatment was done in order to smoothen the gold surface and the remove any oxides.

A PGSTAT20 potentiostat (ECO Chemie, The Netherlands) controlled by GPES 4.9 software package running on a Pentium II computer (Eknadata) was used to record the voltammetric curves. Measurement of the solution $\mathrm{pH}$ was done using an Orion Benchtop pH-meter model 420A. Before each experiment, pure nitrogen was bubbled through the cell solution for $20 \mathrm{~min}$.

The Co(II) tetrasulphonated phthalocyanine sodium salt (CoTSPc) was purchased from Rhodes University of Grahamstown, Eastern Cape (South Africa).

The scanning micro SR-XRF experiments were performed at Beam Line L of the DORIS-III storage ring, HASYLAB (Hamburg, Germany) [14]. This beamline is dedicated to micro-XRF experiments using either white or monochromatic bending magnet excitation and mono- or polycapillary focusing, with routinely available beam sizes of 10-30 $\mu \mathrm{m}$. Fig. 1(a) shows the experimental arrangement used during our scanning micro-XRF experiments, which also allowed optional X-ray absorption near edge structure (XANES) spectroscopic measurements in fluorescence and/ or in transmission mode. A typical XRF-spectrum measured at this instrument for a modified gold electrode corresponding to a measuring time of $500 \mathrm{~s}$ is presented in Fig. 1(b).

The primary X-ray beam is generated by a $1.2 \mathrm{~T}$ bending magnet source which, given the machine energy of $4.465 \mathrm{GeV}$, provides a polychromatic spectral distribution with a critical energy of $16.6 \mathrm{keV}$. After initial low-energy filtering and collimation, the beam was monochromatized by a $\operatorname{Si}(111)$ fixed-exit monochromator $\left(\Delta E / E \approx 10^{-4}\right)$ and further collimated by a motorized cross-slit system. After monochromatization the beam was focused by a monolithic polycapillary half-lens (X-ray Optical Systems Inc., USA), designed specifically for parallel primary beams hence suitable for the focusing of low-divergence synchrotron beams. A microbeam size of about $20 \mu \mathrm{m}$ (FWHM) was obtained at the excitation energy range of $7.7-8.0 \mathrm{keV}$, which was used to excite selectively the Co $\mathrm{K}_{\alpha, \beta}$ fluorescent-lines without exciting $\mathrm{Au}-\mathrm{L}$ lines from the (spectroscopically infinitely thick) gold substrate. The nominal working distance of the polycapillary optic was $5 \mathrm{~mm}$.
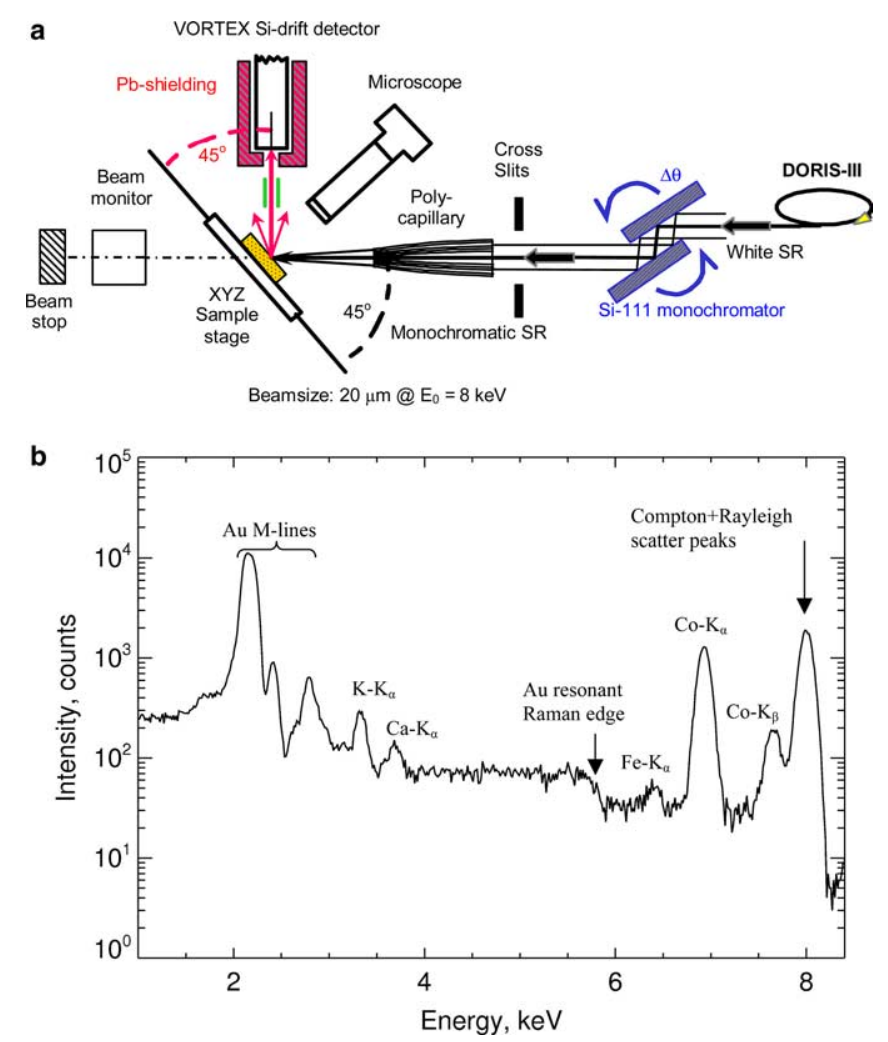

Fig. 1. Schematic representation of the micro SR-XRF instrument installed at HASYLAB Beam Line L (Hamburg, Germany) (a) and a typical XRF-spectrum measured at this instrument for a gold electrode corresponding to a measuring time of $500 \mathrm{~s}(\mathrm{~b})$. 


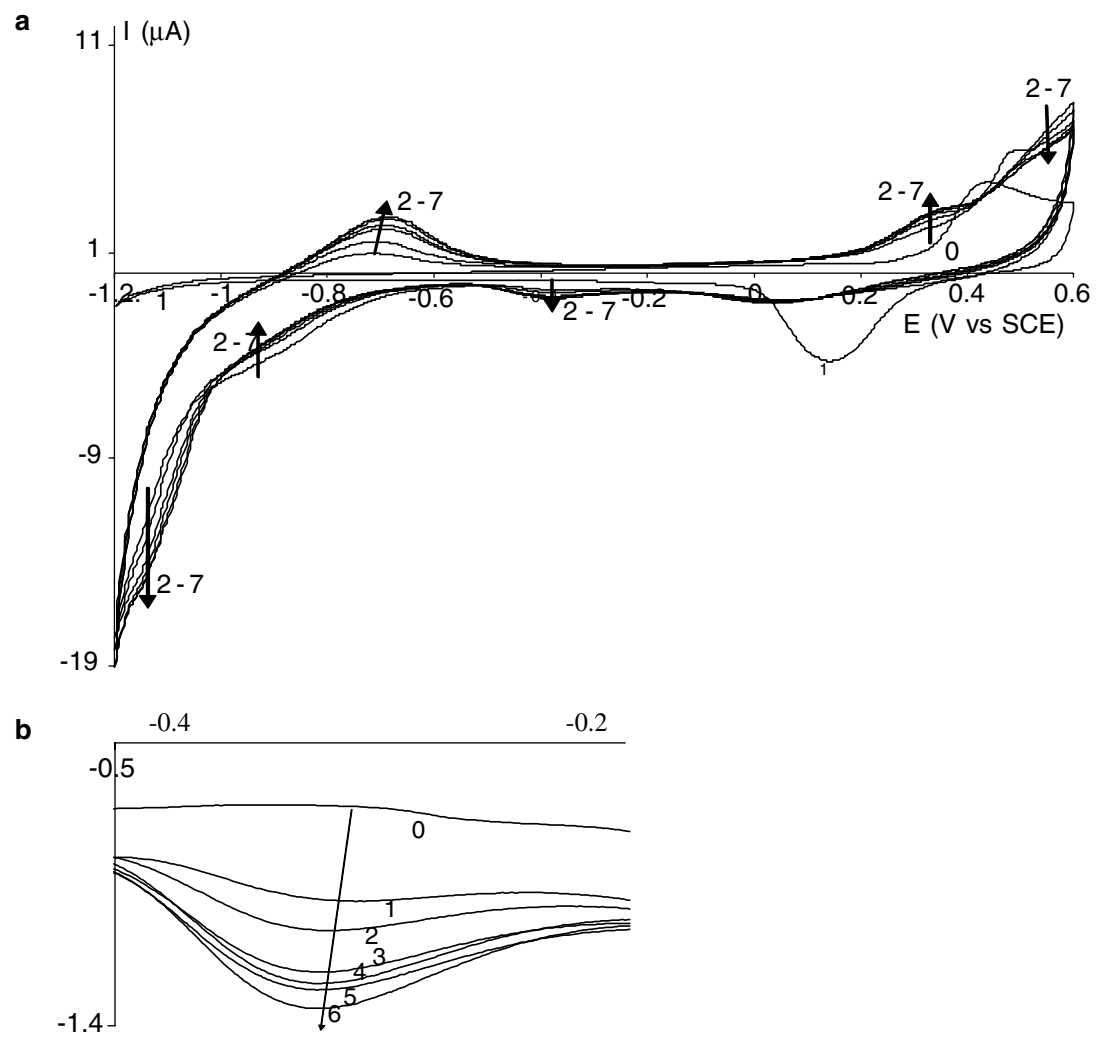

Fig. 2. (a) Current potential curves recorded at a gold electrode with a scan rate of $50 \mathrm{mV} \mathrm{s}^{-1}$ before (1) and after modification (2-7) with a subsequent number of cyclic voltammetric scans in a pH 12 buffer solution containing $6 \mathrm{mmol} \mathrm{L}^{-1} \mathrm{Co}$ (II)TSPc. Number of modification scans are (2) 3 , (3) 5 , (4) 10 , (5) 17, (6) 30 and (7) 100. (b) shows an enlarged view of the reduction process at $-0.38 \mathrm{~V}$ vs. SCE.

Table 1

First three columns show the electrochemical data, respectively, the number of modification scans in a $\mathrm{pH} 12$ buffer solution containing 6 mmol $\mathrm{L}^{-1}$ CoTSPC, the charge due to the $\mathrm{Co}(\mathrm{III}) / \mathrm{Co}(\mathrm{II})$ reduction peak and the calculated surface concentration

\begin{tabular}{lllrll}
\hline $\begin{array}{l}\text { Number of } \\
\text { voltammetric scans }\end{array}$ & Charge $(\mu \mathrm{C})$ & $\begin{array}{l}\text { Surface concentration }\left(\mu \mathrm{g} \mathrm{cm}^{-2}\right) \\
\text { measured by voltammetry }\end{array}$ & $\left\langle I_{\mathrm{Co}}\right\rangle$ & $\delta_{\mathrm{Co}} / \bar{I}_{\mathrm{Co}}(\%)$ & $\begin{array}{l}\text { Surface concentration }\left(\mu \mathrm{g} \mathrm{cm}{ }^{-2}\right) \\
\text { measured by SR-XRF }\end{array}$ \\
\hline 5 & $3.3 \pm 0.2$ & 0.100 & 201.0 & 18.9 & $0.17 \pm 0.03$ \\
10 & $3.8 \pm 0.2$ & 0.115 & 663.8 & 10.5 & $0.44 \pm 0.05$ \\
17 & $3.9 \pm 0.2$ & 0.118 & 511.3 & 16.0 & $0.38 \pm 0.06$ \\
30 & $3.5 \pm 0.3$ & 0.106 & 1404.1 & 7.7 & $1.03 \pm 0.08$ \\
100 & $3.9 \pm 0.2$ & 0.118 & 2036.7 & 31.6 & $3.30 \pm 1.00$ \\
\hline
\end{tabular}

The last three columns show the SR-XRF data, respectively, the average cobalt intensity, its relative standard deviation and the cobalt surface concentration.

Experimental difficulty of the CoTSPc thin-film uniformity studies included the determination of low-amounts of Co (ca. $0.1-0.5 \mathrm{pg}$, assuming a $20 \mu \mathrm{m}$ incident beam) in the presence of partially overlapping Compton and $\mathrm{Au}$ $\mathrm{X}$-ray resonant Raman peaks. Interference with the latter could be significantly reduced by applying an excitation energy well below the Au-L3 absorption edge $\left(E_{0}=8 \mathrm{keV}\right)$, which not only reduces the intensity of Au resonant Raman peaks, but also shifts them below the position of $\mathrm{Co}-\mathrm{K}$ lines (see Fig. 1(b)).

During the CoTSPc uniformity studies $21 \times 21$ or $41 \times 41$ scanning XRF maps were recorded with typically $20 \mu \mathrm{m}$ step-size, using a data collection time of 10-20 s per data point. Absolute detection limits for Co were estimated to be $7-8 \mathrm{ng} \mathrm{cm}^{-2}$ using a measuring time of $20 \mathrm{~s}$. In order to determine the level of uniformity of the deposited CoTSPs thin-film, the scanning and subsequent data reduction strategy described by Kempenaers et al. [15] has been applied.

\section{Results and discussion}

In this study, an electrochemical potential cycling method is used to deposit a film of phthalocyanines onto a gold electrode. This technique enables one to control and characterize the formed layer by following the potential and current during the deposition process as a function of the applied number of voltammetric scans. The modification was done by recording successive cyclic voltammetric scans in a potential window from -1.2 to $0.6 \mathrm{~V}$ vs. SCE 
$\left(50 \mathrm{mV} \mathrm{s}^{-1}\right.$ ) during 100 scans in a $\mathrm{pH} 12$ buffer solution containing $6.00 \mathrm{mmol} \mathrm{L}^{-1} \mathrm{Co}(\mathrm{II}) \mathrm{TSP}$. Fig. 2 shows some of the 100 cyclic voltammetric scans subsequently and continuously recorded at a gold electrode in order to create an electrochemically modified CoTSPc gold electrode. A detailed explanation of all electrochemical processes can be found in the literature $[12,13]$. Only the reduction peak at $-0.38 \mathrm{~V}$ vs. $\mathrm{SCE}\left(\mathrm{Co}(\mathrm{III}) \mathrm{TSP}_{\mathrm{ads}} / \mathrm{Co}(\mathrm{II}) \mathrm{TSP}_{\mathrm{ads}}\right)$ is rele-
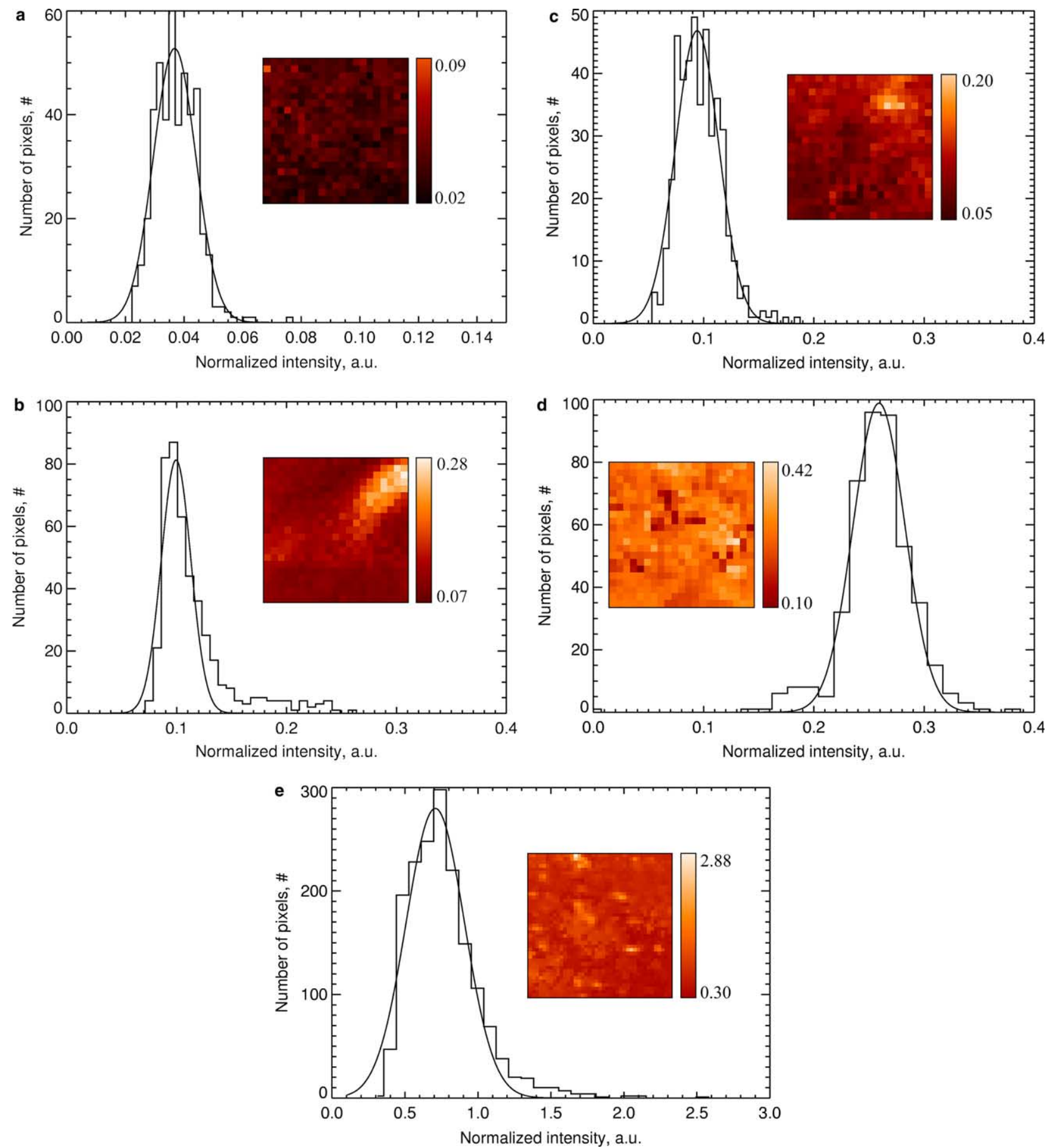

Fig. 3. Elemental map of deposited Co and the corresponding histogram of the detected Co Ka line obtained from a modified gold electrode as a function of scan number: 5 scans (a), 10 scans (b), 17 scans (c), 30 scans (d) and 100 scans (e). The XRF maps consist of a $21 \times 21$ or $41 \times 41$ raster using $20 \mu \mathrm{m}$ step size in both $X$ and $Y$ direction. vant for this study. The charge related to this adsorption process, which first increases with growing number of voltammetric scans, becomes constant around scan 17. This scan is also called the breaking point, the scan characterized by a maximum adsorption. Before the breaking resulting in a chaotic multilayer of CoTSPc molecules. Once the saturation of deposited CoTSPc is obtained (scan point, the adsorption process is kinetically controlled 


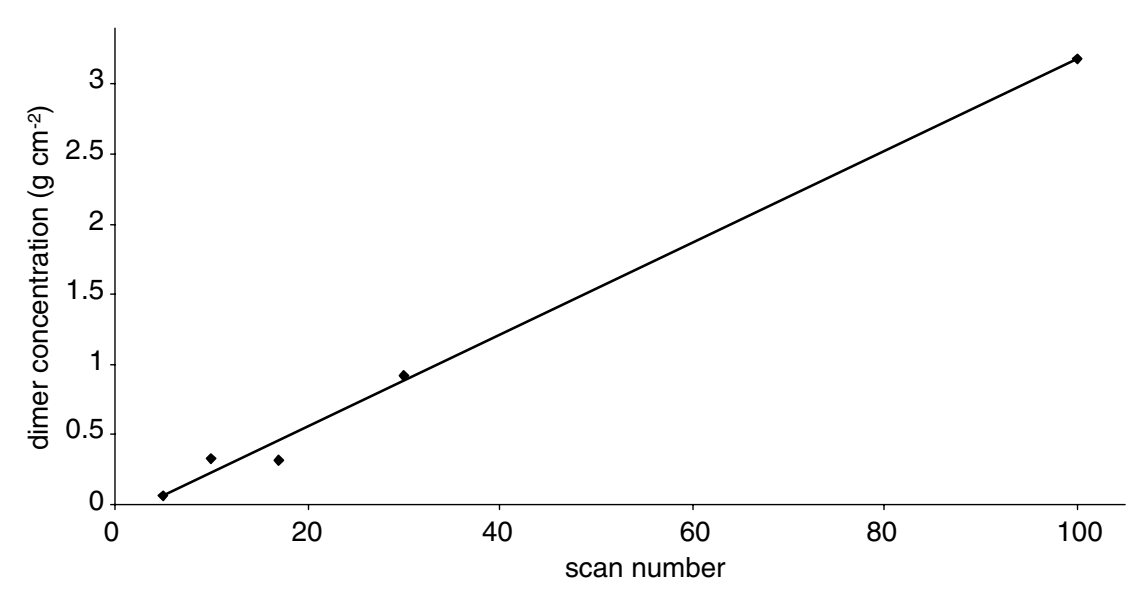

Fig. 4. Relationship between dimer concentration and number of voltammetric scans.

17), a reorganization of the chaotic multilayer takes place towards a more stable and ordered multilayer structure of CoTSPc molecules (columnar aggregates) $[12,13]$.

Table 1 shows the derived charges related to the $\mathrm{Co}(\mathrm{III})_{\mathrm{ads}} / \mathrm{Co}(\mathrm{II})_{\mathrm{ads}}$ reduction, after a background correction, indicating the increase of the charge with growing number of voltammetric scans before the breaking point and the stabilization after scan 17 . The cobalt surface concentrations calculated from the electrochemical data as well as those derived from the XRF measurements are presented. The XRF measurements were performed after different number of scans. The elemental maps of the deposited $\mathrm{Co}$ and the corresponding histograms of the detected Co Ka intensities (Fig. 3(a) scan 5, (b) scan 10, (c) scan 17, (d) scan 30 and (e) scan 100), derived from the SR-XRF measurements after normalization using the gold $\mathrm{M}$ lines are presented in Fig. 3. The average cobalt intensity, its relative standard deviation and the average cobalt surface concentration is summarized in Table 1.

These Figs. 3(a)-(e) not only provide evidence of the occurrence of adsorbed CoTSPc but also give an estimate of the amount of adsorbed CoTSPc. First, the surface concentrations based on the XRF data are significant higher than those obtained from the electrochemical data. Secondly, in contrast with the electrochemical data, it can be seen that the amount of cobalt keeps increasing even after scan 17. Based on these observations, the discrepancy between both methods can be explained by the fact that only CoTSPc monomer species and not dimers can be electrochemically detected. As only monomer species adsorb onto the gold surface by potential cycling [16], a constant value for the charge is expected after scan 17, even when dimers are also stacked around the adsorbed monomers. It is expected that CoTSPc dimers surround the modified electrode in any circumstances, but these compounds are not electrochemically active. In contrast, the XRF measurements are able to detect the total amount of both cobalt species, monomers as well as dimers. Therefore, the XRF signal which includes both the monomer as well as the dimer signals leads to significantly higher surface concentration of cobalt compared to the electrochemically detectable amount. The difference between the cobalt amount calculated from the XRF measurements (monomers and dimers) and the one obtained from the electrochemical experiments (only monomers) gives an estimate of the amount of dimers. It can be seen in Fig. 4 that the number of CoTSPc dimers stacked around the modified electrode increases with scan number. This means the adsorbed monomer layer, surrounded with CoTSPc dimers, builds up continuously resulting in an increased value for the Co signal.

\section{Conclusion}

This paper gives a detailed study of the morphology and heterogeneity of the deposited phthalocyanine layer onto a gold electrode. The deposition was done by successive cyclic voltammetric scans in a phthalocyanine solution. A non-destructive characterization of CoTSPc deposited on a gold electrode was performed by means of synchrotron $\mathrm{X}$-ray fluorescence as a function of the applied number of voltammetric scans. The electrochemical measurements and scanning micro SR-XRF allowed us to derive the adsorbed Co surface concentration values.

Only monomer CoTSPc species could be observed with electrochemical techniques. In contrast, XRF detects both the monomer as well as dimer species leading to significantly higher amounts of detected cobalt compared to values derived from electrochemical techniques. The difference between the Co surface concentration values obtained by the above techniques gives an estimate of the amount of CoTSPc dimers.

\section{Acknowledgments}

This work was supported by the European Community - Research Infrastructure Action under the FP6 "Structuring the European Research Area" Programme (through the Integrated Infrastructure Initiative "integrating Activity on Synchrotron and Free Electron Laser Science"). Ghent University (BOF) financed this work. 


\section{References}

[1] C.C. Leznoff, A.B.P. Lever, Phthalocyanines Properties and Applications, vol. 3, VCH Publishers, Weinheim, 1993.

[2] A.B.P. Lever, Chemtech 17 (1987) 506.

[3] M. Kato, Y. Nishioka, K. Kaifu, K. Kawamura, S. Ohno, Appl. Chem. Lett. 86 (1985) 196.

[4] J.E. Kuder, J. Imag. Sci. 32 (1988) 51.

[5] T.A. Temofonte, K.F. Schoch, J. Appl. Chem. 65 (1989) 1350.

[6] K.J. Balkus, M. Eissa, R. Levado, J. Am. Chem. Soc. 117 (1995) 10753.

[7] A. Andreev, V. Ivanova, L. Prahov, I.D. Schopov, J. Mol. Catal. 95 (1995) 197.

[8] K. De Wael, P. Westbroek, E. Temmerman, Electroanalysis 17 (3) (2005) 263.

[9] R.A. Durst, A.J. Bäumer, R.W. Murray, R.P. Buck, C.P. Andrieux, Pure Appl. Chem. 69 (1997) 1317.
[10] W.O. Kutner, J. Wang, M. L'Her, R.P. Buck, Pure Appl. Chem. 70 (1998) 1301

[11] H.O. Finklea, in: R.A. Meyers (Ed.), Encyclopedia of Analytical Chemistry, vol. 11, Wiley, New York, 2000.

[12] K. De Wael, P. Westbroek, E. Temmerman, J. Electroanal. Chem. 567 (2004) 167.

[13] K. De Wael, P. Westbroek, P. Bultinck, D. Depla, P. Vandenabeele, A. Adriaens, E. Temmerman, Electrochem. Commun. 7 (2005) 87.

[14] G. Falkenberg, O. Clauss, A. Swiderski, Th. Tschentscher, X-Ray Spectrom. 30 (3) (2001) 170.

[15] L. Kempenaers, K. Janssens, L. Vincze, B. Vekemans, A. Somogyi, M. Drakopoulos, A. Simionovici, F. Adams, Anal. Chem. 74 (2002) 5017.

[16] K. Peeters, K. DeWael, L. Vincze, A. Adriaens, Anal. Chem. (in press). 